\title{
Philonsorbonne
}

15 | 2021

Année 2020-2021

\section{Les bienfaits politiques de l'ignorance : les pauvres et l'économie du savoir chez Bernard Mandeville et Jacques Necker}

Jordan MESSERLÉ

\section{OpenEdition}

Journals

Édition électronique

URL : https://journals.openedition.org/philonsorbonne/1856

DOI : 10.4000/philonsorbonne.1856

ISSN : 2270-7336

Éditeur

Publications de la Sorbonne

Édition imprimée

Date de publication : 1 janvier 2021

Pagination : 137-151

ISSN : 1255-183X

Référence électronique

Jordan MESSERLÉ, «Les bienfaits politiques de l'ignorance : les pauvres et l'économie du savoir chez Bernard Mandeville et Jacques Necker », Philonsorbonne [En ligne], 15 | 2021, mis en ligne le 03 février 2021, consulté le 08 juin 2021. URL : http://journals.openedition.org/philonsorbonne/1856 ; DOI : https://doi.org/10.4000/philonsorbonne.1856 


\title{
Les bienfaits politiques de l'ignorance : les pauvres et l'économie du savoir chez Bernard Mandeville et Jacques Necker
}

\author{
Jordan MESSERLÉ
}

\section{Introduction - De la nature de l'esprit à la nature de l'ordre social}

Je souhaiterais souligner que le XVIII ${ }^{\mathrm{e}}$ siècle est le foyer d'un renversement dans l'intrication de ce que nous avons appelé « théorie de la connaissance » et «théorie politique ». Les recherches sur le fonctionnement de l'esprit pouvaient, jusqu'alors, servir d'instruments critiques contre l'essentialisation des différences sociales. En insistant sur l'unicité de la nature humaine, elles élucidaient l'artificialité de préjugés qui érigeaient des barrières infranchissables entre groupes sociaux (par exemple, les hommes et les femmes chez les auteurs dits «philogynes ») ${ }^{1}$. Si la différenciation des conditions sociales ne procède d'aucun ancrage naturel, alors l'histoire - individuelle ou collective - pouvait apparaitre comme la matrice des causes contingentes auxquelles les inégalités doivent être reconduites. L'éducation ou le triomphe historique d'un groupe sur un autre deviennent ainsi les sources de la différenciation des esprits et des distinctions. D'où la multiplication des projets de " généalogies » ou d'enquêtes historiques pour dévoiler l'artifice derrière les prétendues vérités intangibles du monde social.

Toutefois, certains auteurs du XVIII ${ }^{\mathrm{e}}$ siècle, dont Bernard Mandeville et Jacques Necker, posent le problème en des termes différents. Tout en acceptant l'unicité de la nature humaine, et une homogénéité - au moins relative - des facultés cognitives, ils justifient un inégal accès à la connaissance au nom des lois de fonctionnement de l'ordre social. Il ne

1. Voir la contribution de Clément Raymond dans ce dossier : «L'usage féminin du savoir : plaisir de l'étude et émancipation chez Poulain de la Barre et Émilie du Châtelet ». 
s'agit plus de déduire, ou de critiquer, un ordre social à partir d'une théorie de l'esprit, mais de proposer une théorie de la différenciation des esprits et de l'inégal accès au savoir, à partir des principes qui assurent la prospérité de l'association politique. Or pour ces auteurs, l'ignorance des pauvres semble une conséquence, certes regrettable, mais nécessaire, de la structure des sociétés modernes.

Mandeville, dans la première moitié du XVIII ${ }^{\mathrm{e}}$ siècle, Necker dans la seconde, ne prétendent à la réviviscence d'aucune différence essentielle entre groupes sociaux. En revanche, leur description du fonctionnement optimal des corps politique fixe les limites souhaitables à la diffusion sociale des connaissances. En ce sens, leur réponse à la question « qui peut savoir ? » est corrélée au degré d'inégalité qu'ils jugent essentiel au corps politique : l'ignorance est sans remède car elle appartient intrinsèquement au meilleur des mondes social possible. Paradoxalement, l'inégal accès à la connaissance se trouve de nouveau naturalisé, mais la différenciation des esprits repose désormais sur les règles inhérentes à une société bien ordonnée.

Mandeville soutient ainsi que l'on ne peut prétendre aux plaisirs des sociétés modernes, sans accepter l'exclusion d'une classe de ces jouissances et de la diffusion du savoir. Contre l'inconséquence philanthropique, qui soulage sa conscience en prétendant œuvrer à l'instruction des pauvres, il faudrait accepter que la division du travail devienne la seule ordonnatrice de l'accès des esprits à l'éducation.

Necker considère, quant à lui, qu'une association politique fondée sur la protection du droit de propriété ne peut manquer de produire une classe laborieuse ignorante. Toutefois, il s'interroge sur la compatibilité de ce manque de lumières avec la permanence du lien social. D'où le rôle politique qu'il confère à un terme médian entre l'ignorance et le savoir : l'opinion.

Le rapprochement de ces deux auteurs repose en ce sens moins sur une filiation intellectuelle que sur le partage d'une forme d'argumentation commune: celle qui envisage l'ignorance du pauvre comme un mal nécessaire auquel les sociétés ne peuvent remédier sans mettre en péril leur prospérité économique ou leur cohésion. Précisons que dans les écrits de Mandeville et de Necker étudiés, le terme «pauvre» se réfère au «travailleur pauvre ", c'est-à-dire à l'individu dont le travail permet à peine de satisfaire les besoins vitaux. En ce sens, cette contribution exclut d'autres figures de la pauvreté comme le mendiant ou le vagabond. De même, le terme « ignorance », tel que l'emploient ces auteurs, n'est pas neutre axiologiquement. Il renvoie au manque d'éducation des classes laborieuses relativement aux classes lettrées et «policées" (sinon à l'aristocratie intellectuelle et financière à laquelle appartiennent les Necker). L'ignorance n'implique donc pas de dénier aux pauvres des savoir pratiques ou empiriques qui, par exemple, naissent de la transmission ou de l'exercice d'un métier'.

2. Ces connaissances jouent, pour Mandeville, un rôle non négligeable dans l'évolution des sociétés et la découverte de nouvelles techniques. Voir Renee Prendergast, «Knowledge, innovation and emulation in the evolutionary thought of Bernard Mandeville », Cambridge, Journal of Economics, 2014, vol. 38, n 1, p. 87-107. 


\section{I. « Toute société bien ordonnée suppose une dose d'ignorance » (Bernard Mandeville)}

\section{(a) Théorie de la nature humaine et hiérarchie des conditions}

Qu'est-ce que l'homme pour la Fable des abeilles ${ }^{3}$ ? Un être matériel, « composé de passions, qui toutes tant qu'elles sont, à mesure qu'elles sont excitées et prennent la première place, le gouvernent tour à tour qu'il le veuille ou non ${ }^{4}$. Un des thèmes bien connus de la Fable consiste alors à montrer que le procès de civilisation ne repose sur aucune qualité morale propre à la nature humaine, mais sur une habile domestication de ses passions ${ }^{5}$. L'analyse de ce soubassement passionnel permet à Mandeville d'interpréter l'histoire de la formation des sociétés politiques, et d'élucider les mécanismes psychologiques à l'origine de leur prospérité économique moderne. Or, cette dernière laisse peu de place à la vertu véritable : elle repose sur des désirs égocentrés qui apparaissent comme les véritables ressorts du fonctionnement social. Néanmoins, si les passions et les désirs sont les maîtres-mots de la mécanique générale des corps politiques, il ne s'ensuit pas que les hommes se déterminent selon les mêmes passions dominantes. En ce sens, il convient de comprendre comment se spécifient les passions à l'échelle individuelle afin de déterminer si l'anthropologie mandevillienne suppose, ou non, une prédisposition au savoir.

Mandeville considère que les hommes présentent tous, par nature, un même spectre de passions. Toutefois, ils ne les expriment pas au même degré : toutes choses égales, la «structure interne » de l'individu détermine celles qui présenteront le plus de poids dans la formation de son caractère. Autrement dit, l'individuation psychologique repose en partie sur une base physiologique que Mandeville reconduit à la notion médicale de «tempérament ${ }^{6}$. Certes, il n'est pas certain que Mandeville définisse

\footnotetext{
3. La première édition de la Fable paraît anonymement en 1714. Elle comporte alors le poème La ruche mécontente, ou les coquins devenus honnête, les Recherches sur l'origine de la vertu morale, et vingt remarques. Ce n'est qu'avec sa réédition en 1723 et ses deux additions, l'Essai sur la charité et les écoles de charité et la Recherche sur la nature des sociétés, que le texte de Mandeville rencontre un véritable succès de scandale. Sur la réception de cette seconde édition, outre l'introduction de Lucien et Paulette Carrive de l'édition Vrin, voir notamment Jonathan Brody Kramnick, "Unwilling to be short, or plain, in any thing concerning gain": Bernard Mandeville and the Dialectic of Charity », The Eighteenth Century, 1992, vol. 33, n 2, p. 148-175.
}

4. Bernard Mandeville, La Fable des abeilles, Première Partie, traduction de Lucien et Paulette Carrive, Paris, Vrin, 2010 (désormais Fable I), Introduction, p. 41.

5. En particulier de l'amour de soi et l'orgueil dans la première partie de la Fable. Voir notamment la Recherches sur la nature de la société qui répond en 1723 à l'idée de sociabilité naturelle défendue par Shaftesbury (ses Charasteristics of men, manners, opinions, times sont publiés en 1711), et les Recherches sur l'origine de la vertu morale qui proposent une genèse de la façon dont l'homme sauvage a été dompté pour devenir l'homme moderne policé.

6. «[...] par quoi on entend le mélange ordonné ou désordonné des fluides du corps» (Fable I, remarque R, p. 163). Sur le rapport de Mandeville à la science médicale de son temps, voir E.J. Hundert, The Enlightenment's Fable. Bernard Mandeville and the Discovery of Society, Cambridge, Cambridge University Press, 1994, p. 35-49. 
clairement la part relative qui revient au tempérament dans la genèse du caractère individuel. Néanmoins, il affirme sans ambiguïté que cette détermination, inscrite dans les spécificités corporelles de l'individu, compose nécessairement avec d'autres causes exogènes (notamment l'habitude et l'éducation) ${ }^{7}$.

Lorsqu'il évoque la notion de tempérament, Mandeville prend donc soin de préciser que ses analyses ne valent que ceteris paribus : elles écartent méthodologiquement les causes sociales qui participent à la construction de la personnalité ${ }^{8}$. Lorsque le médecin britannique précise le rôle de cet ancrage physiologique dans le développement des facultés individuelles, il rejette ainsi l'utilisation du tempérament pour fonder l'infériorité cognitive des femmes relativement aux hommes ${ }^{9}$. Une véritable attention aux déterminations de nos facultés devrait, selon lui, plutôt conduire à embrasser l'idée inverse : celle d'une supériorité naturelle des capacités "féminines », que nous masquent l'éducation et le rôle social qui échoient généralement aux femmes ${ }^{10}$. Autrement dit, les facultés cognitives des femmes sont avant tout les produits des normes contingentes de leur socialisation, et non du développement nécessaire de leur constitution.

Si le « tempérament» permet de rendre compte de certaines singularités comportementales (le héros, le martyr ${ }^{11}$ ou, inversement, le pleutre pathologique), il ne définit donc pas un cadre rigide. Des causes exogènes amendent nécessairement le développement de la constitution naturelle. Ainsi, s'il existe un courage inscrit dans les organes, l'expérience montre aussi que même les hommes aux constitutions les moins fermes s'endurcissent au spectacle de la mort, et deviennent "artificiellement» courageux $^{12}$. À partir de ce modèle, on ne peut donc prétendre à aucune

7. « Je crois que la différence de dons entre les hommes dépend, sans autre cause, de la différence qui est entre eux, soit dans la structure même, c'est-à-dire dans la composition plus ou moins exacte de leur construction [frame], soit dans l'usage qu'ils en font " (Fable, Deuxième Partie, traduction de Lucien et Paulette Carrive, Paris, Vrin, 2007 [désormais Fable II], quatrième dialogue, p. 142-143). Je souligne.

8. Pour un exemple théorique de différenciation individuelle fondée sur le tempérament (toutes choses égales), voir Fable II, Troisième dialogue, p. 98.

9. Voir Fable II, Quatrième dialogue, p. 146-147.

10. D'où la place séminale qu'occupe Mandeville dans les réflexions des Lumières anglaises sur les femmes selon Karen O'Brien. Les ambiguïtés du médecin britannique quant à ce qui tient du physiologique ou de l'incorporation de normes sociales seraient matricielles pour les débats postérieurs sur la condition féminine (Karen O'Brien, Women and Enlightenment in Eighteenth-Century Britain, Cambridge, Cambridge University Press, 2009, notamment p. 19-27). Pour une synthèse et la mise en contexte de la pensée mandevillienne sur les femmes, voir Maurice Marks Goldsmith, "'The Treacherous Arts of Mankind' : Bernard Mandeville and Female Virtue », History of Political Thought, 1986, Vol. 7, № 1, p. 93-114.

11. Voir Fable I, Remarque R, p. 165-166. Mandeville ironise sur les discours qui prétendent que les martyrs de la foi n'ont pu endurer leur supplice qu'avec un soutien surnaturel. Ces discours se montrent «ignorants de la force de notre nature et ne savent pas qu'il y a des hommes d'un tempérament résolu qui peuvent s'exciter jusqu'à l'enthousiasme sans autre aide que des passions violentes » (p. 165).

12. Fable I, Remarque R, p. 165. 
différence naturelle entre les enfants pauvres et riches; en revanche, leurs conditions d'existence induisent, elles, un développement différencié de leurs facultés. Pour Mandeville, l'enfant, lorsqu'il est l'objet continuel de l'attention de ses parents ou de ses nourrices, a davantage l'occasion de développer son esprit que le nouveau-né abandonné à lui-même car ses parents sont pris dans la nécessité vitale du travail ${ }^{13}$. Une telle théorie rend donc en droit les pauvres éducables au même titre que les riches en reconduisant la différenciation des esprits à des causes matérielles. Certes, cette éducation produirait des effets sensiblement différents selon la constitution des enfants ${ }^{14}$, mais un habile instructeur pourrait, si les conditions matérielles étaient égales, indifféremment tirer parti des passions juvéniles pour motiver l'investissement dans l'étude ${ }^{15}$.

Seules les inégalités des sociétés modernes semblent donc vouer aux gémonies un projet d'éducation universel ou, du moins, celui d'une large diffusion du savoir. Toutefois, le problème ne se pose pas en ces termes chez le médecin britannique qui refuse toute transformation de la structure sociale. De façon symptomatique, la "supériorité naturelle» des femmes ne donne, chez lui, lieu à aucune revendication de droits politiques. Mais la tension entre sa théorie de la différenciation des esprits et la préférence donnée à une segmentation sociale rigide, apparaît aussi de façon spectaculaire dans le cas des «génies ». Mandeville admet la naissance ponctuelle d'individus dotés de facultés cognitives hors du commun. Mais, quelle que soit l'étendue de ces facultés, il rejette tout devoir de la société de cultiver ces talents lorsqu'ils apparaissent chez les pauvres :

Je sais qu'on m'objectera toujours qu'il y a de la barbarie à ce que les enfants pauvres n'aient pas la possibilité d'exercer leurs forces du moment que Dieu ne leur a pas refusé des talents et du génie naturels plus qu'aux riches. Mais je n'arrive pas à croire que cela est plus injuste que le fait qu'ils n'aient pas d'argent du moment qu'ils ont le même désir de dépenser que les autres ${ }^{16}$.

13. Fable II, quatrième dialogue, p. 159-160. Mandeville, tout en acceptant le tempérament comme principe de différenciation des individus, raisonne à partir d'un cadre empiriste. Il insiste tout particulièrement sur l'importance de parler aux enfants pour développer leur esprit. Or, les parents pauvres ne peuvent consacrer à l'interaction avec leur progéniture un temps égal à celui des classes libérées du labeur (ou, du moins, qui disposent de l'argent nécessaire pour déléguer ce soin à des nourrices). Si bien que l'on " attribue souvent à une incapacité naturelle ce qui est entièrement dû à l'omission de cette instruction première » (p. 160).

14. « Il y a des gens qui ne peuvent jamais apprendre la musique, et feront pourtant de bons mathématiciens, d'autres qui joueront parfaitement du violon et qui seront des ânes toute leur vie, quels que soient les gens qu'ils fréquenteront » (Fable I, Remarque R, p. 163).

15. Notamment en la présentant comme une source d'estime sociale. Dans la première partie de la Fable, Mandeville moque ceux qui «veulent que tout ce qui est bénéfique à la société naisse d'un bon principe » et « attribuent l'effet de l'émulation sur les écoliers à une vertu de l'esprit» (Fable I, Remarque N, p. 110). Ces professeurs de vertu refusent en réalité de voir que l'envie et le désir de gloire jouent un rôle fondamental dans l'étude. Sur l'emploi de la honte et de l'orgueil dans l'éducation, voir encore Fable II, Deuxième dialogue, p. 72-73.

16. Essai sur la charité et les écoles de charité, dans Fable I, p. 242. 
La nature de l'esprit ne fonde, à elle seule, aucun accès de droit à l'instruction. Ce refus d'instruire le génie pauvre n'est que le pendant épistémique d'une logique d'exclusion économique propre aux sociétés marchandes. Or ce cas limite est généralisable pour Mandeville qui prétend rationaliser l'exclusion des pauvres du savoir au nom des principes de la prospérité sociale.

\section{(b) Les conditions économiques de la prospérité}

La justification de l'ignorance du pauvre ne se comprend qu'au sein d'une situation historique précise des sociétés humaines. En effet, leur insertion dans un réseau d'échanges international et la généralisation de la division du travail engendrent une production sans précédent de biens de consommation $^{17}$. Tournées vers le commerce et la production, ces sociétés offrent à leurs membres une aisance matérielle jusqu'alors inégalée, et leurs permettent de profiter des « douceurs les plus raffinées de l'existence ${ }^{18}$.

Toutefois, Mandeville considère que cette aisance a pour condition l'existence d'une classe laborieuse qui accomplit les tâches les plus ingrates qu'occasionne la division du travail. Cette classe ne peut (et ne doit) pas, en outre, jouir de ces productions nouvelles, faute de salaires suffisants. Les raisons de cette exclusion de la consommation moderne se trouvent dans l'appréhension « mercantiliste ${ }^{19}$ » des échanges de Mandeville : la prospérité économique de la nation repose avant tout sur sa balance commerciale. Or la concurrence internationale dans l'exportation des biens implique de produire à bas coût, et donc de maintenir la rémunération du travail le plus bas possible (le travail n'est considéré que comme un coût, et Mandeville ne

17. Rappelons que Mandeville écrit dans une période d'importante expansion économique. L'augmentation des échanges maritimes en nombre et en volume à partir de la moitié du $\mathrm{XVII}^{\mathrm{e}}$ siècle rend accessibles au marché anglais de nouveaux biens à prix modéré.

18. Fable I, Préface, p. 24.

19. Le concept de mercantilisme, en tant que construction rétrospective de l'histoire de la pensée économique, pose bien évidemment d'épineux problèmes méthodologiques (voir Céline Spector, « Le concept de mercantilisme », Revue de métaphysique et de morale, $\mathrm{n}^{\circ} 39$, 2003, p. 289-309). Il permet néanmoins d'éviter la téléologie, qui ferait de Mandeville un précurseur brouillon de Smith, en soulignant la dette de sa pensée sociale envers le faisceau d'idées économiques qui caractérise le $\mathrm{XVII}^{\mathrm{e}}$ et le début du XVIII ${ }^{\mathrm{e}}$ siècle. Le médecin britannique souscrit ainsi sans réserve à l'idée d'une prospérité nationale liée à des exportations qui l'emportent en valeur sur les importations (ce qui justifie des mesures protectionnistes). En revanche, l'adhésion à cette idée s'accompagne de la défense, peu " mercantiliste », d'un luxe relatif et du refus de considérer que seul le stock d'argent importe pour définir la puissance d'une nation (voir l'exemple de l'Espagne dans la Fable I, Remarque Q, p. 151-152). Pour une lecture (certes méthodologiquement périlleuse) de Mandeville à la frontière théorique d'un mercantilisme finissant et d'un capitalisme naissant, voir Paulette Carrive, "Mandeville, le mercantilisme et le capitalisme », dans La Pensée politique anglaise, PUF, 1994, p. 301-322. 
donne aucune importance économique spécifique à la consommation des classes laborieuses ${ }^{20}$ ).

Mais la prospérité économique des sociétés marchandes semble alors reconduite à des fondements fragiles. Elle apparaît suspendue au consentement - paradoxal - des travailleurs pauvres à un destin social qui les prive de « douceurs » qu' ils participent pourtant à produire ou à importer. Or Mandeville propose deux moyens pour assurer l'attachement de la classe laborieuse à l'ordre social : faire en sorte que chacun puisse subsister par le travail, et compter sur les effets psychologiques de l'ignorance.

Le premier moyen suppose d'éviter un déséquilibre entre taille de la population et demande de travail. Le politique, s'il n'a pas vocation à garantir un hypothétique droit au travail, se doit néanmoins d'encourager les principales sources de cette demande: l'industrie et l'agriculture. L'augmentation relative des rendements agricoles ou de l'exploitation des ressources piscicoles par rapport à la demande permet, en outre, une diminution du prix des biens de subsistance et donc, pour Mandeville, la possibilité d'une baisse des salaires ${ }^{21}$. En conséquence :

Le grand art donc, de faire le bonheur d'une nation et de lui donner ce qu'on appelle la prospérité, consiste à fournir à tout le monde l'occasion d'avoir du travail. Pour y parvenir, le premier soin du gouvernement doit être de favoriser toute la vérité des manufactures, des arts et des métiers que l'ingéniosité humaine est capable d'inventer; et son deuxième soin d'encourager l'agriculture et la pêche dans toutes leurs branches, afin que la terre entière se voie forcée aux mêmes efforts que les hommes ${ }^{22}$.

Les raisons de la participation des pauvres à un ordre économique dont les fruits ne leur sont pas destinés s'expliquent encore par les effets de l'ignorance. Mandeville soutient en effet qu'il existe un contentement ${ }^{23}$ parfaitement compatible avec leur travail acharné, mais il suppose de penser l'ignorance comme le voile utile qui dissimule au pauvre le malheur auquel

20. Mandeville ironise dans la dernière remarque de le Fable sur les accusations qui le présentent comme l'apologiste d'un luxe sans limite. Il précise alors que ce luxe ne vaut pas pour les pauvres, et qu'il a «posé comme maximes inviolables qu'il faut tenir strictement les pauvres au travail, et qu'il est sage de soulager leur nécessité, mais fou de les en guérir [...]» (Fable I, Remarque Y, p. 191).

21. «[...] il faut favoriser l'agriculture et la pêche dans toutes leurs branches afin que les victuailles et par conséquent le travail soient bon marché » (Fable I, Remarque Y, p. 191). Du moment que la demande de travail n'excède pas l'offre, l'étalon des salaires du travail est le prix des biens de subsistance.

22. Fable I, Remarque Q, p. 153. Mandeville précise peu après cet extrait que « c'est de cette politique, et non point d'insignifiantes réglementations de la profusion et de la frugalité (qui quoi qu'on fasse suivront leur cours selon la condition des gens) qu'il faut attendre la grandeur et le bonheur des nations » (loc. cit.).

23. «Par contentement [content] j'entends cette calme sérénité d'esprit dont jouissent les hommes qui se croient heureux et sont satisfaits de leur condition » (Fable I, Remarque V, p. 186). 
la prospérité économique moderne le condamne. Si l'éducation a tant de pouvoir sur la maturation des passions, alors, réciproquement, Mandeville espère que son absence - couplée à l'habitude du labeur dès le plus jeune âge - limite l'orgueil à un stade embryonnaire. C'est à cette condition que l'on peut, selon lui, imaginer le pauvre laborieux heureux ${ }^{24}$.

\section{(c) L'ignorance, condition du contentement}

L'Essai sur la charité et les écoles de charité développe longuement ce thème: il est irrationnel de prétendre éclairer les enfants des classes laborieuses alors que le fonctionnement économique des sociétés modernes les astreint à des tâches difficiles et répétitives. La virulence de l'attaque de Mandeville contre l'éducation charitable est, en ce sens, un procès en inconséquence. Les prétendus bienfaiteurs de l'humanité, lorsqu'ils entendent éclairer le peuple, refusent d'admettre que leur projet est dénué de sens au sein de sociétés dont la prospérité dépend d'une pauvreté structurelle. En effet, l'éducation populaire est le foyer de deux effets pervers: non seulement elle prive la production d'une force de travail disponible, mais elle décourage aussi le travailleur d'accomplir sa tâche.

Il y a une abondance de travail pénible et sale à faire, et il faut accepter une vie grossière. Où trouverons-nous meilleure pépinière de ces êtres nécessaires que parmi les enfants des pauvres ? Personne à coup sûr n'en approche davantage et n'en est plus propre. En outre, ce que j'ai appelé des choses pénibles ne paraît ni n'est tel pour ceux qui y ont été élevés et qui ne connaissent pas autre chose $\mathrm{e}^{25}$.

Puisque l'existence d'une classe de pauvres est une condition à la production des plaisirs modernes, il faut se garder de dégoûter le pauvre du travail en l'instruisant de vues étrangères à sa destinée sociale. Or le vœu philanthropique d'une éducation populaire ne peut qu'éveiller des passions qui conduisent les enfants de la classe laborieuse à considérer avec mépris leur condition :

\footnotetext{
24. Des commentateurs pensent, ici, voir une contradiction entre le postulat d'un égal partage des passions humaines et leur étouffement relatif chez le pauvre au nom de la prospérité sociale (voir Francesca Pongiglione et Mikko Tolonen, « Mandeville on charity schools : happiness, social order and the psychology of poverty ", Erasmus Journal for Philosophy and Economics, Volume 9, Issue 1, 2016, p. 82-100). Les ambiguïtés de Mandeville sur le sujet ne font pas de doute. En revanche, l'ampleur de cette «contradiction » dépend de la force que l'on donne à l'éducation et à l'habitude dans le développement des passions : les pauvres seront, certes, naturellement « orgueilleux », mais le seront-ils suffisamment, et auront-ils une connaissance suffisante du mode de vie des autres classes, pour prendre conscience de leur malheur?
}

25. Essai sur la charité et les écoles de charité dans Fable I, p. 243. 
Ceux qui ont passé une grande partie de leur jeunesse à apprendre à lire, à écrire et à calculer, s'attendent, non sans justice, à avoir un emploi où ces talents leur serviront; en général, ils regarderont avec un total mépris le travail véritable, je veux dire le travail accompli au service des autres dans la plus basse condition sociale et pour la plus misérable rémunération ${ }^{26}$.

L'éducation multiplie des désirs qui s'accordent mal avec les réquisits de la production moderne. D'une part, le pauvre éduqué, mais contraint aux travaux pénibles, se condamne à la frustration et à l'auto-apitoiement. D'autre part, Mandeville précise que la qualité de son travail s'en ressent nécessairement: l'éducation cultive un orgueil qui cause un désinvestissement chez le pauvre conscient de l'injustice de sa condition, et donc une baisse de la productivité de son travail.

Le projet de Mandeville dans l'Essai sur la charité et les écoles de charité se résume alors en une phrase provocatrice : "prouver la nécessité qu'il y a d'une certaine dose d'ignorance dans une société bien ordonnée ${ }^{27}$. Tout comme l'opulence et la prospérité sont indissociables du rôle social du vice, on ne peut assurer le fonctionnement de l'ordre économique sans établir les conditions de la résignation de la classe laborieuse à la pauvreté.

\section{La propriété, l’ignorance et le lien social (Jacques Necker)}

Dans la seconde moitié du siècle, Necker constitue en apparence un négatif de Mandeville. Loin de la réputation scandaleuse de l'auteur de la Fable des abeilles, il fut un ministre populaire dont on retenait l'opposition aux mesures de libéralisation du commerce des grains au nom de la subsistance du peuple. Ses écrits témoignent, en outre, d'une conscience aiguë des rapports de domination qu'accentuaient les transformations économiques de la France de la deuxième moitié du XVIII ${ }^{\mathrm{e}}$ siècle. Comme Mandeville, il admet pourtant que les sociétés modernes sont fondées sur une inégalité irréductible, qui condamne une partie de la population à un labeur inconciliable avec l'étude. Mais à la différence du médecin anglais, Necker, acteur et témoin de la Révolution, se soucie de la contradiction possible entre ignorance et pauvreté du peuple d'une part, et maintien de la cohésion sociale d'autre part. L'ignorance de la classe laborieuse recoupe, en ce sens, une réflexion plus générale sur les conditions du lien social au sein de sociétés fortement inégalitaires. 


\section{(a) L'ignorance, loi sociale}

Necker, même s'il ne s'embarrasse pas d'une théorie générale de la nature humaine, admet parfaitement l'hypothèse d'une faculté de connaître également partagée. Selon lui, "la faculté de savoir et d'entendre est un don général de la nature $»^{28}$, et il est vain de se quereller a priori sur les capacités cognitives de membres de telle ou telle classe. Certes, les esprits semblent plus ou moins facilement « perfectibles $»^{29}$, mais cette perfectibilité différenciée répond du seul hasard de la nature et n'est le privilège d'aucun groupe social.

En revanche, l'actualisation de cette capacité de savoir dépend de la place qu'occupe l'individu au sein de sociétés dont l'inégalité dans la distribution de la propriété est généralement la règle. Pour Necker, cette distribution définit, en effet, le temps que chacun peut consacrer au développement de ses facultés :

[...] si les propriétés étaient égales, chacun travaillerait modérément, et chacun saurait un peu, parce qu'il resterait à chacun une portion de temps à donner à l'étude et à la pensée; mais dans l'inégalité des fortunes, effet de l'ordre social, l'instruction est interdite à tous les hommes nés sans propriétés, car toutes les subsistances étant entre les mains de la partie de la nation qui possède l'argent ou les terres, et personne ne donnant rien pour rien, l'homme né sans aucune ressource que sa force, est obligé de la consacrer au service des propriétaire, dès le premier moment où elle se développe, et de continuer ainsi toute sa vie $[\ldots]^{30}$.

L'ordre social moderne consacre une homologie entre répartition de la propriété et opportunité de perfectionner son esprit. Le pauvre, dès que ses capacités physiques sont suffisamment développées, est contraint à un labeur qui exclut le temps de l'étude.

Certes, selon ce même principe, une répartition plus égalitaire de la propriété permettrait à chacun de cultiver son esprit. Mais Necker raisonne à partir de l'horizon théorique d'un droit de propriété qui, malgré les abus

28. Jacques Necker, Sur la législation et le commerce des grains (1775), chap. XXIV, dans Euvres Complètes de M. Necker, publiées par M. le Baron de Staël, son petit-fils (désormais O.C.), Tome I, Paris, 1820-1821, p. 130.

29. Necker mobilise la "perfectibilité » dans les Réflexions philosophiques sur l'égalité afin de rendre compte de la formation nécessaire d'inégalités sociales. Il accuse ainsi les systèmes égalitaristes de violer les lois de la nature humaine, puisqu'ils nient cette tendance des esprits à se différencier, et donc de l'individu à pouvoir occuper un rang en corrélation avec leur génie (voir O.C., Tome X, Paris, 1820-1821, p. 358 et p. 492). Chez Necker, comme chez tous les défenseurs libéraux de la propriété du siècle suivant, cet argument est hautement paradoxal puisqu'il ne vaut que «toutes choses égales ». Or Necker ne cesse de répéter par ailleurs que la propriété induit nécessairement des distinctions matérielles qui interdisent à chacun de développer également son esprit.

30. Sur la législation et le commerce des grains, O.C., T. I, chap. XXIV, p. 130. 
qu'il engendre, ne souffre aucune contestation radicale ${ }^{31}$. Deux arguments principaux expliquent la sanctification de ce droit. D'une part, il conditionne la prospérité économique des sociétés modernes en entretenant un désir d'appropriation qui aiguillonne l'activité économique. D'autre part, le droit de propriété est le motif à l'origine des sociétés civiles, et la justice de l'association politique se définit alors en fonction de sa capacité de le protéger. À ce titre, Necker mobilise à plusieurs reprises l'expérience de pensée de la position originelle pour justifier l'accord universel qui devrait prévaloir sur sa légitimité. Supposons de purs esprits, ignorants des positions sociales qu'ils occuperont au sein de la société, ces derniers admettraient nécessairement la convenance du droit de propriété avec l'ordre social ${ }^{32}$. Le peuple, bien qu'il soit pris dans le réseau de dépendances qu'occasionne la concentration inégale de la propriété, se proposerait donc un remède pire que le mal en lui portant atteinte. Il tarirait la principale source de prospérité sociale, et conférerait à l'État un pouvoir tyrannique contraire au fondement de l'association politique ${ }^{33}$.

Une fois admises la nécessité de la protection de la propriété privée et son inégale répartition, l'ignorance du peuple en découle donc, pour Necker, comme une « loi sociale »" Mais de là naît aussi une difficulté redoutable : si la société engendre implacablement l'ignorance du peuple, alors il ne peut disposer des lumières suffisantes pour entendre les justifications rationnelles d'une propriété dont il est exclu. En un sens, une part non négligeable de la réflexion politique de Necker cherche à résoudre un même paradoxe : certes, les sociétés naissent de l'ambition de protéger le droit de propriété, mais

31. Albert Soboul souligne le décalage entre l'«audace critique » de Necker et son « timide réformisme » qui aboutit à des conclusions conservatrices (voir « Lumières, critiques sociales et utopie pendant le XVIII ${ }^{\mathrm{e}}$ siècle français », dans Jacques Droz (dir.), Histoire générale du socialisme, Tome I, Paris, PUF, 1997, p. 175).

32. Nous parlerions, aujourd'hui, de « voile d'ignorance ». Voir De l'importance des opinions religieuses, O.C., T. XII, p. 35 et les Réflexions philosophiques sur l'égalité où Necker reprend un modèle proche à partir de l'exemple de la loterie: O.C., T.X, p. 417 et p. $460-461$.

33. Levons une ambiguïté : si Necker prétend faire du droit de propriété un fondement de l'association politique, comment expliquer qu'il se range, lors de la querelle des blés, du côté des auteurs appelés «prohibitifs » par leurs adversaires ? Comment expliquer qu'il justifie une limitation de la liberté du commerce des grains ? Il n'est pas certain que l'on puisse apporter une réponse absolument satisfaisante à cette tension, mais l'on peut rappeler (a) que Necker considère qu'il est rationnel d'admettre des aménagements lorsque le droit de propriété contrevient à d'autres principes de l'organisation sociale - dont la survie des associés. Par conséquent, si les propriétaires des blés « réunissent en eux [...] les droits généraux de la propriété, qui ne mettent aucune borne à leurs prétentions », ils détiennent aussi des "devoirs attachés au dépôt d'une denrée essentielle à la vie » (Sur la législation et le commerce des grains, O.C., T. I, quatrième partie, chap. VI, p. 297). (b) En outre, un abus circonstancié de la liberté du commerce risque d'attirer la colère du peuple contre la propriété en général et, à ce titre, des limites à l'exportation est un prix dérisoire à payer pour des propriétaires qui profitent de l'essentiel de l'ordre juridique (ibid., p. 207).

34. Sur la législation et le commerce des grains, O.C., T. I, Première partie, chap. XXIV, p. 129. 
ce dernier menace de dissoudre l'association en produisant des inégalités qui rendent impossible toute adhésion générale à sa justification. Ce hiatus confronte ainsi les corps politiques au risque constant de rébellion des classes laborieuses. Celles-ci ne peuvent, pour Necker, comprendre les subtilités de théodicées sociales dont on leur répète, pourtant, qu'elles justifient « rationnellement» leur condition.

\section{(b) Une métaphysique sociale impuissante}

On peut donc comprendre l'indignation qu'ont suscitée certaines thèses, à rebours des grandes idées du siècle, du texte Sur la législation et le

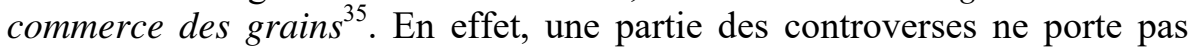
tant sur les analyses économiques de l'opuscule que sur son rejet du pouvoir de la raison à fonder le lien social. Condorcet, par exemple, accepte sans réserve la sanctification du droit de propriété et les conséquences de l'inégalité matérielle dans la formation des salaires. En revanche, il n'admet pas que l'on dénie aux pauvres la capacité de convenir rationnellement de la justice du droit de propriété et de la nécessité de son respect absolu ${ }^{36}$.

A fortiori, le paradoxe que prétendait voir Necker se heurtait à l'ambition d' " éclairer » le peuple, afin de réaliser le miracle de la fusion d'intérêts que l'ordre économique semblait, en apparence du moins, dissocier. Telle était la solution physiocratique, dont les représentants s'étaient faits les champions des projets d'éducation populaire ${ }^{37}$. Ces derniers considèrent la diffusion de la connaissance économique comme un moyen pour rendre manifeste la nature de la solidarité qui unit des acteurs économiques partageant un même intérêt : l'augmentation du produit net ${ }^{38}$. De sorte que les projets éducatifs des physiocrates reposent sur un double postulat:

(1) Cognitif: il existe une connaissance possible d'un ordre naturel de la formation et de la distribution des richesses accessible à l'ensemble des esprits. Ce qu'ils appuient sur la notion gnoséologique d'évidence, c'est-à-

35. Diderot, qui fréquentait le salon de Mme Necker, oppose au futur directeur général des finances un modèle concentrique de diffusion de l'opinion : le savoir philosophique présente bien un effet social en se propageant de proches en proches. Voir, Denis Diderot, «Lettre du 10 juin 1775 à Necker », dans Correspondance, Tome V, Paris, Robert Laffont, 1997.

36. Voir la Lettre d'un laboureur de Picardie à $M$. $N^{* * *}$ et les Réflexions sur le commerce des blés (dans CEuvres de Condorcet publiées par A. Condorcet O'Connor et M.-F. Arago, T. XI, Paris, Firmin Didot Frères, 1847).

37. Les références ne manquent pas (même si l'éducation n'a jamais été le souci de Quesnay). Citons notamment la proposition de précis d'éducations qui se spécifient selon chaque classe de Mirabeau (dans les Lettres sur la législation ou l'ordre légal, dépravé, rétabli et perpétué, Tome III, Berne, 1775), ou encore l'ouvrage de Lemercier de la Rivière De l'instruction publique, ou considérations morales et politiques sur la nécessité, la nature et la source de cette instruction (Stockholm, 1775).

38. Voir Philippe Steiner, «Le projet physiocratique : théorie de la propriété et lien social », Revue économique, 1987, volume 38, nº 6, p. 1111-1128. 
dire cette «tendance naturelle» qui convertit universellement les esprits à l'ordre naturel une fois chassées les opinions infondées ${ }^{39}$.

(2) Psychologique : la connaissance de cet ordre induit le consentement des membres des différentes classes économiques au rôle qui leur échoit dans la production richesses. En éclairant le fonctionnement de l'ordre social et l'absence d'alternatives, l'éducation et la liberté de la presse confortent les hommes dans leur sentiment d'appartenir à l'édifice politique qui optimise le bonheur de chacun, même si ce bonheur ne peut se traduire par des situations matérielles égales.

Or Necker rejette précisément l'optimisme des physiocrates envers la capacité de l'éducation à opérer la conversion heureuse des classes laborieuses à l'ordre qu'ils prétendent déceler. La connaissance ne constitue pas une consolation contre les maux des pauvres dont, au contraire, elle ne montre que trop bien la nécessité. Les physiocrates s'illusionneraient finalement sur la force de la raison à provoquer un assentiment nécessaire. Selon lui, les lois naturelles des philosophes économistes valent, hypothétiquement, pour le législateur, mais : « [...] elles ne sauraient frapper de la même manière l'homme jeté sur la terre, sans bien, sans ressource et sans espérance; et il ne rendra jamais un hommage libre à la beauté d'un ensemble où il n'y a pour lui que laideur, abjection et mépris $\gg$.

Selon Necker, il n'y pas de lien nécessaire entre instruction et cohésion sociale. Il reprendra d'ailleurs l'objection contre les thuriféraires de l'égalité et d'une morale laïque. Ces derniers, en prétendant déduire rationnellement les droits et devoirs des hommes en société dans des «catéchismes» républicains, sont coupables de conférer à la raison des pouvoirs qu'elle ne possède pas. Ils attendent trop de la diffusion sociale de la connaissance, et oublient une caractéristique essentielle de la bonne politique : elle suppose une obéissance que la raison seule ne suffit pas à assurer. Puisque, en effet, «l'habitude du respect et de la subordination ne peut jamais être un simple produit de la réflexion $\gg$.

\section{(c) Magie sociale}

Mais l'ignorance, entendue comme seule privation de savoir, manque elle-même d'une positivité qui motiverait l'adhésion volontaire des pauvres à l'ordre social. Le politique ne peut donc se contenter des effets de l'ordre économique : il doit convertir l'esprit des membres des classes laborieuses à la société qu'il administre.

39. Paul-Pierre Lemercier de la Rivière, L'Ordre naturel et essentiel des sociétés politiques, Londres, 1767, chap. IX, p. 61.

40. De l'importance des opinions religieuses, O.C., Tome XII, p. 24-25.

41. Réflexions philosophiques sur l'égalité, O.C., Tome X, p. 351. 
D'où l'appel répété de Necker, contre les projets républicains, à ne pas abandonner les différences statutaires héritées de l'Ancien Régime. Le consentement à la structure inégalitaire des sociétés implique, selon lui, d'entretenir l'illusion d'une différence de nature entre les positions sociales. La reconnaissance institutionnelle de rangs permet que le rapport normal des pauvres « au spectacle de l'oisiveté, de l'abondance et du bonheur apparent des riches » relève d'une forme de " magie ${ }^{42}$ qui naturalise des distinctions sociales contingentes. En ce sens, il incombe à la législation d'entretenir la perception légitime d'un monde social hiérarchisé. Puisque l'on ne se compare qu'entre égaux, cette opinion « magique » qui masque les origines artificielles des inégalités devient la condition de possibilité de la félicité - et de la soumission - des pauvres, et donc de leur attachement à l'ordre social ${ }^{43}$.

Pour Necker, les révolutionnaires s'abusent lorsqu'ils prêchent la liquidation de l'architecture statuaire d'Ancien Régime. Quand bien même on abolirait les rangs pour proclamer une égalité des droits, il demeurerait un clivage économique entre riches et pauvres, entre propriétaires et peuple démuni, qui condamnerait toujours une partie de la population à l'ignorance. Certains réformateurs rêveraient d'un résultat contradictoire: consacrer le gouvernement républicain et faire de l'éducation la panacée de la conciliation des intérêts sans amender un ordre économique qui condamne une classe au strict nécessaire. Or :

Ce n'est $[\ldots]$ pas le despotisme des gouvernements, c'est l'empire de la propriété qui réduit le sort de la grande partie des hommes au plus étroit nécessaire. Cette loi de dépendance existe d'une manière à peu près égale sous les différents genres d'autorité politiques; et partout le salaire des ouvrages qui n'exigent aucune éducation est soumis aux mêmes proportions ${ }^{44}$.

Pour Necker, la conciliation de l'égal accès au savoir, du droit de propriété et de la cohésion sociale, est un idéal inaccessible à toute théorie sociale. Entendue l'illégitimité de toute loi attentatoire à la propriété privée, Necker envisage alors le rôle social d'un terme médian entre l'ignorance et la diffusion du savoir : l'opinion. À la veille de la Révolution, dans $D e$ l'importance des opinions religieuses, il plaide ainsi pour reconsidérer son rôle politique : le dogme, qui se passe de commentaires, est politiquement plus utile pour consolider le lien social que les arguties d'une morale laïque qui risquent, toujours, de prêter le flanc à la critique et donc d'échouer à « convertir» les esprits à l'ordre social ${ }^{45}$.

42. Sur la législation et le commerce des grains, O.C., T. I, Première partie, chap. XXIV, p. 127.

43. «Il faut bien se garder d'ailleurs de retenir autour des idées spéculatives les hommes destinés par leur situation aux travaux mécaniques; car il importe à leur bonheur qu'ils en contractent l'habitude dès l'enfance » (Réflexions philosophiques sur l'inégalité, O.C., T. X, p. 375).

44. Du pouvoir exécutif dans les grands états, O.C., T. VIII, p. 485.

45. Sur les alternatives à la proposition de Necker d'ériger l'opinion religieuse en ciment du lien social, voir Bertrand Binoche, Religion privée, opinion publique, Paris, Vrin, 2011. 
Qui souhaite sauver la propriété privée et concilier les intérêts antagonistes qui règnent au sein l'association politique, se trouve donc nécessairement reconduit à l'utilité politique du théologique, sinon de la superstition $^{46}$. Comme le redécouvrira face à la question sociale une partie, au moins, de la pensée libérale de la première moitié du XIX ${ }^{\mathrm{e}}$ siècle, il ne reste plus qu'à espérer que les opinions religieuses motivent la charité et suffisent à inspirer une résignation heureuse. Le socialisme naissant proposera, lui, une autre solution au paradoxe neckérien: si République il y a, elle ne peut être que « sociale».

\section{Conclusion}

De quoi ces discours sur l'ignorance des pauvres sont-ils le symptôme ? Pour reprendre une formule chère à Rousseau, elles résolvent, à leur manière, les « contradictions du système social» qui deviennent apparentes aux auteurs du XVIII ${ }^{\mathrm{e}}$ siècle. L'histoire des sociétés modernes semble entée sur des inégalités qu'il devient de plus en plus difficile de fonder en raison. Sans doute faut-il tenter de les mettre en "harmonie», comme l'on commence à répéter sans relâche, mais le secret de ces consonances se dérobe malgré les entreprises nouvelles de théodicée sociale. En particulier, l'accès différencié au savoir ne peut être reconduit à une théorie de la nature humaine : les « bons esprits » appartiennent en puissance à toutes les classes. Pourtant, les suites à donner à cette communauté de nature et, bientôt, à l'idée d'un partage égal de droits fondamentaux, n'ont rien d'une évidence. L'émerveillement devant les progrès des arts et de l'industrie, devant le confort nouveau des sociétés modernes, présente sa part d'ombre dans son besoin - sinon sa production - de travailleurs ignorants. Ferguson l'avance froidement: "l'ignorance est la mère de l'industrie aussi bien que la superstition ${ }^{47}$. Dans ce contexte, Mandeville - cette $"$ tête lucide ${ }^{48}-$ et Necker, ont, du moins, le mérite de la conséquence (fût-elle cynique), face à des contradictions auxquelles nous n'avons peut-être pas fini d'essayer d'apporter une réponse.

46. On comprend que Rivarol mette en doute la religiosité de Necker, et l'accuse de simplement développer le vers de Voltaire, «si Dieu n'existait pas, il faudrait l'inventer» (Antoine Rivarol, Première lettre à M. Necker sur l'importance des opinions religieuses, Berlin, 1788, p. 4).

47. Adam Ferguson, Essai sur l'histoire de la société civile, Livre IV, Paris, PUF, 1992, p. 279.

48. Karl Marx, Le Capital, Livre I, chap. XXII, Paris, PUF, 1993, p. 689. 